\title{
Self-tuning Feedforward Control for Active Vibration Isolation of Precision Machines
}

\author{
M.A. Beijen ${ }^{*, * *}$ J. van Dijk ${ }^{* *}$ W.B.J. Hakvoort ${ }^{* *, * * *}$ \\ M.F. Heertjes * \\ * Eindhoven University of Technology, Department of Mechanical \\ Engineering, PO Box 513, 5600 MB Eindhoven, The Netherlands \\ (e-mail: M.A.Beijen@tue.nl). \\ ** University of Twente, Department of Mechanical Engineering, PO \\ Box 217, 7500 AE Enschede, The Netherlands \\ *** Demcon Advanced Mechatronics, Institutenweg 25, $7521 \mathrm{PH}$ \\ Enschede, The Netherlands
}

\begin{abstract}
A novel feedforward control strategy is presented to isolate precision machinery from broadband floor vibrations. The control strategy aims at limiting the low-frequency controller gain, to prevent drift and actuator saturation, while still obtaining optimal vibration isolation performance at higher frequencies. This is achieved by limiting the low-frequency control action such that almost no phase shift is introduced at higher frequencies. To minimize model uncertainties, the feedforward controller is implemented as a self-tuning IIR filter that estimates the parameters online. Only a few parameters have to be estimated, which makes the algorithm computationally efficient. An additional feedback controller is designed to make the self-tuning algorithm more robust. The effects of feedforward and feedback control add up. The control strategy is successfully validated on an experimental setup of a vibration isolator.
\end{abstract}

Keywords: Active vibration isolation, feedforward control, self-tuning, semi-conductor industry.

\section{INTRODUCTION}

Vibration isolators are widely used in high-precision machines, e.g. wafer scanners, see Tjepkema et al. (2011). Passive vibration isolators consist of physical springs and dampers between the floor and the supported machine. Such isolators can only attain a limited performance because a large passive damping of the suspension mode leads to less vibration isolation at high frequencies (Karnopp and Trikha (1969)). Active vibration isolators contain an additional control system with sensors and actuators to further improve the vibration isolation performance. Feedback control is widely used for vibration isolators, examples are Zuo et al. (2004); Tjepkema et al. (2011). However, the performance of feedback controllers is limited by the controller bandwidth and the Bode sensitivity integral.

An alternative approach for active vibration isolation is causal feedforward control, see for example Van der Poel (2010); Landau et al. (2011). In contrast with feedback control, where the measured machine motion is used as controller input, causal feedforward control uses measurements of external vibrations (e.g. floor vibrations) as controller input. In general, due to the causal nature of the disturbance measurement, the performance of the feedforward control at high frequencies is limited by the feedforward sensitivity integral, see Heertjes et al. (2013). Moreover, modeling errors limit the performance, since the feedforward control force is calculated using a system model. Furthermore, it is undesired to use feedforward control for low-frequency disturbances, as this can easily lead to drift and actuator saturation. However, using regular high-pass filters to limit low-frequency controller gains, leads to a phase shift in the controller that deteriorates the performance.

In this paper, a novel feedforward control strategy is presented for broadband disturbance rejection. The lowfrequency controller gain is limited without introducing a large phase shift at higher frequencies, such that at higher frequencies optimal performance is obtained. To minimize model uncertainties and compensate for parameter variations in time, the parameters are estimated online using a self-tuning IIR filter with $n^{\text {th }}$ order basis functions, resulting in an $n^{\text {th }}$-order roll-off in the transmissibility function. An IIR filter with first-order basis functions is proposed in Williamson and Zimmermann (1996); Yuan (2007). Compared with self-tuning FIR filters (see e.g. Van der Poel (2010)), IIR filters give the designer more freedom because poles can be placed at locations other than $z=0$. Therefore, a computationally efficient algorithm is obtained because the system can be accurately described with only a few coefficients. Fixing the poles in the basis functions prevents instability due to pole adaptation. Moreover, it results in a linear formulation of the estimation problem such that a unique solution exists. Feedback control is added to make the convergence process of the self-tuning filter more robust, and to show that the effects of feedforward and feedback control add up.

The remainder of this paper is organized as follows. A model for the vibration isolator is given in Section 2 and a simple feedback control law in Section 3. In Section 4, a fixed-gain feedforward controller is derived, while in 
Section 5 the step to self-tuning controllers is made. In Section 6, an experimental validation is presented.

\section{MODEL DESCRIPTION}

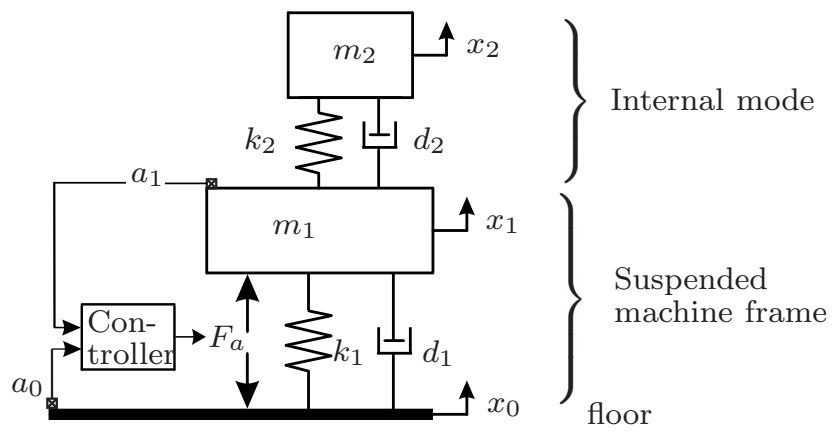

Fig. 1. Model of an active vibration isolator.

Consider the system shown in Fig. 1 . Masses $m_{1}=5.4 \mathrm{~kg}$ and $m_{2}=3.9 \mathrm{~kg}$, connected by the spring $k_{2}=1400 \mathrm{kN} / \mathrm{m}$ and the viscous damper $d_{2}=20 \mathrm{Ns} / \mathrm{m}$, represent a simplified precision machine with one dominant internal mode. The machine is suspended by a hybrid mount having a stiffness of $k_{1}=160 \mathrm{kN} / \mathrm{m}$ and a viscous damping of $d_{1}=80 \mathrm{Ns} / \mathrm{m}$. Using these system parameters, a suspension mode at $20 \mathrm{~Hz}$ and an internal mode at $125 \mathrm{~Hz}$ are obtained. These frequencies are representative for an active hard mount vibration isolator, see Tjepkema et al. (2011). Actuator force $F_{a}$ is used for active vibration isolation. Accelerometers on the floor $\left(a_{0}\right)$ and the machine $\left(a_{1}\right)$ are used for measurement and control. Since precision machines are usually placed on stiff and heavy floors, the mechanical coupling between $F_{a}$ and $x_{0}$ is neglected. To deal with systems having mechanical coupling, the reader is referred to Landau et al. (2011).

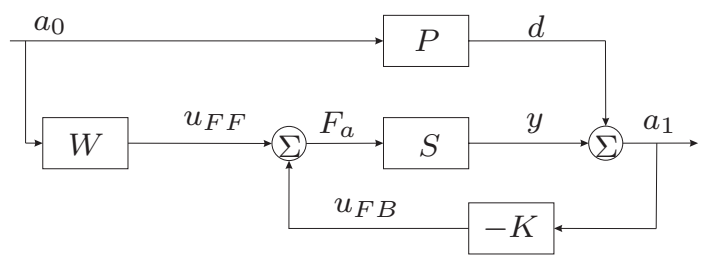

Fig. 2. Combined feedforward/feedback control scheme for active vibration isolation.

Active isolation of floor vibrations is obtained by a combination of feedforward control and feedback control. The controller scheme is shown in Fig. 2. The input disturbance signal is denoted by $a_{0}$ and the error signal is denoted by $a_{1}$. The latter is formed by two signals: a signal $d$ that is caused by the disturbance from the primary path $P$, i.e. the passive system, and a component $y$ that is caused by the control action from the secondary path $S$. The control action $F_{a}$ is the sum of the feedback controller output $u_{F B}$ and the feedforward controller output $u_{F F}$, computed by the controllers $K$ and $W$, respectively.

The primary path $P$ is described by the transfer function from floor vibrations to machine vibrations. The Laplace variable $s$ is used to represent $P$ in the frequency domain,

$$
P(s)=\left(d_{1} s+k_{1}\right) \frac{n_{r}(s)}{p_{r}(s)},
$$

with $n_{r}(s)=\left(m_{2} s^{2}+d_{2} s+k_{2}\right)$ describing an antiresonance at $\omega_{A R}=\sqrt{k_{2} / m_{2}}$ due to the internal mode, and $p_{r}(s)$ describing the poles of the suspension mode and the internal mode as

$$
\begin{aligned}
p_{r}(s)= & \left(m_{1} s^{2}+d_{t o t} s+k_{t o t}\right)\left(m_{2} s^{2}+d_{2} s+k_{2}\right) \\
& -\left(d_{2} s+k_{2}\right)^{2},
\end{aligned}
$$

with $d_{t o t}=d_{1}+d_{2}$ and $k_{t o t}=k_{1}+k_{2}$. Given that $d_{1}, d_{2}$ are small and $k_{2} \gg k_{1}$, it can be derived that the poles described by $p_{r}(s)$ correspond with a suspension mode at $\omega_{\text {susp }}=\sqrt{k_{1} /\left(m_{1}+m_{2}\right)}$ and an internal mode at $\omega_{R}=\sqrt{\left(1+m_{2} / m_{1}\right) k_{2} / m_{2}}$.

The secondary path $S$ is described by the transfer function from control action to machine vibrations. In the frequency domain, $S$ is given by

$$
S(s)=s^{2} \frac{n_{r}(s)}{p_{r}(s)} .
$$

The total machine motion is given by

$$
\begin{aligned}
A_{1}(s)= & P(s) A_{0}(s)+S(s) F_{a} \\
= & P(s) A_{0}(s)+S(s) W(s) A_{0}(s) \\
& -S(s) K(s) A_{1}(s) .
\end{aligned}
$$

From (4), the transmissibility function $T$ is derived:

$$
T(s)=\frac{A_{1}(s)}{A_{0}(s)}=\frac{P(s)+S(s) W(s)}{1+S(s) K(s)} .
$$

In the remainder of this paper, $T$ will be used as a performance measure for the vibration isolator. Equation (5) shows that $K$ and $W$ can be designed independently. The numerator in (5) describes the reduction obtained by feedforward control, while the denominator describes the reduction by feedback control. Without control $(K=W=$ $0), T$ equals $P$.

\section{FEEDBACK CONTROL}

A feedback controller $K$ is used to add skyhook damping, see Karnopp and Trikha (1969), to the suspension mode and the internal mode. Using $a_{1}$ as input, $K$ is given by

$$
K(s)=\frac{F_{a}(s)}{A_{1}(s)}=\frac{K_{v}}{s+1} .
$$

This controller integrates the acceleration signal using the tame integrator $1 /(s+1)$ to obtain the platform velocity, needed for skyhook damping. $K_{v}=2000 \mathrm{Ns} / \mathrm{m}$ is tuned such that both the suspension mode and the internal mode are sufficiently damped without making the controller bandwidth unnecessarily high. The effect of feedback control is shown in Fig. 3.

\section{FIXED-GAIN FEEDFORWARD CONTROL}

In this section, the fixed-gain feedforward controller is derived. The controller gain is limited at low frequencies, to prevent drift and actuator saturation, without introducing a big phase shift at higher frequencies. Therefore, optimal vibration isolation performance is obtained at higher frequencies. In the initial design, perfect knowledge of the system parameters is assumed. In practice, these parameters are not known exactly, so the actual performance of the fixed-gain controller will deviate. Therefore, the influence of non-perfect parameter estimations is also 
discussed in this section. The fixed-gain controllers give the mathematical relations needed to design the self-tuning controllers in Section 5.

\subsection{The approximate Wiener controller}

From (5) it follows that, regardless of the feedback controller $K$, perfect cancellation of floor vibrations is obtained when using the following feedforward control law,

$$
W_{o p t}(s)=-S^{-1}(s) P(s)=-\frac{1}{s}\left(\hat{d}_{1}+\frac{\hat{k}_{1}}{s}\right),
$$

with $\hat{d}_{1}=d_{1}$ and $\hat{k}_{1}=k_{1}$ being perfect estimates of the suspension damping and stiffness, respectively. In literature, this controller is referred to as the Wiener controller. Note that $W_{o p t}$ does not depend on $n_{r}(s)$ or $p_{r}(s)$, i.e. it needs no prior knowledge of the internal mode. Physically this makes sense, because $m_{2}$ can only be excited via $m_{1}$. So if floor vibrations are not transmitted to $m_{1}$, they will not be transmitted to $m_{2}$ either.

Next, the pure integrators in (7) are replaced by tame integrators to prevent drift and actuator saturation. Therefore the integrating actions are cut off at $\alpha \mathrm{rad} / \mathrm{s}$, leading to

$$
\begin{aligned}
W_{1}(s) & =-\frac{1}{s+\alpha}\left(\hat{d}_{1}+\frac{\hat{k}_{1}}{s+\alpha}\right) \\
& =-\frac{1-\frac{\alpha}{s+\alpha}}{s}\left(\hat{d}_{1}+\frac{1-\frac{\alpha}{s+\alpha}}{s} \hat{k}_{1}\right) .
\end{aligned}
$$

Neglecting feedback control $(K=0)$, and substituting (8) in (5) with perfect estimates $\hat{k}_{1}=k_{1}$ and $\hat{d}_{1}=d_{1}$, the following transmissibility function is obtained:

$$
T(s) \approx 2 \frac{\alpha}{s+\alpha} P(s), \text { for } s=j \omega, \omega>\alpha .
$$

Equation (10) shows that if $\alpha \rightarrow 0$, then $T \rightarrow 0$, resulting again in perfect cancellation of floor vibrations. If $\alpha \neq$ 0 , then a $-20 \mathrm{~dB} /$ decade roll-off is introduced in $T$ for $\omega>\alpha$. This roll-off can be increased, i.e. the vibration isolation properties can be improved, by using higher-order controllers. Define the controller $W_{3}$, in which the firstorder low-pass filters in (9) are replaced by third-order low-pass filters, or

$$
W_{3}(s)=-\frac{1-\left(\frac{\alpha}{s+\alpha}\right)^{3}}{s}\left(\hat{d}_{1}+\frac{1-\left(\frac{\alpha}{s+\alpha}\right)^{3}}{s} \hat{k}_{1}\right)
$$

Using $W_{3}$ and assuming again $\hat{k}_{1}=k_{1}$ and $\hat{d}_{1}=d_{1}$, the transmissibility is given by

$$
T(s) \approx 2\left(\frac{\alpha}{s+\alpha}\right)^{3} P(s), \text { for } s=j \omega, \omega>\alpha .
$$

Compared with (10), (12) has two more poles at $s=-\alpha$. These poles increase the roll-off of $T$ for frequencies $\omega>\alpha$. This observation leads to the following proposition.

Proposition 1. Let $T$ be the transmissibility from floor vibrations to machine vibrations in the context of Fig. 1. For the Wiener controller given by (7), an approximate Wiener controller is given by

$$
W_{n}(s)=-\frac{1-L_{n}(s)}{s}\left(\hat{d}_{1}+\frac{1-L_{n}(s)}{s} \hat{k}_{1}\right),
$$

in which $L_{n}$ may be an arbitrary $\mathrm{n}^{\text {th }}$-order low-pass filter with unity gain, and from which follows that

$$
\begin{aligned}
T(s)= & L_{n}(s)\left(\hat{d}_{1} s+\left(2-L_{n}(s)\right) \hat{k}_{1}\right) \frac{n_{r}(s)}{p_{r}(s)} \\
& +\underbrace{\left(\left(d_{1}-\hat{d}_{1}\right) s+\left(k_{1}-\hat{k}_{1}\right)\right) \frac{n_{r}(s)}{p_{r}(s)}}_{T_{\text {error }}(s)} .
\end{aligned}
$$

In case of perfect estimation, i.e. $\hat{k}_{1}=k_{1}$ and $\hat{d}_{1}=d_{1}$, the error term $T_{\text {error }}$ in (14) vanishes, such that $T$ reduces to:

$$
T(s) \approx 2 L_{n}(s) P(s), \text { for } s=j \omega, \omega>\alpha .
$$

Proof. The proof follows from substitution of (13) in (5), with $K(s)=0$.

Equation (15) shows that with (13) and $\hat{k}_{1}=k_{1}, \hat{d}_{1}=d_{1}$, potentially a transmissibility function with a roll-off of arbitrary dB/decade can be created. As an example, Fig. 3 shows the transmissibilities when $L_{n}$ is a $1^{\text {st }}$-order, $3^{\text {rd }}$ order or $5^{\text {th }}$-order low-pass filter and $\alpha=2 \mathrm{~Hz}$. Note that for frequencies lower than $\alpha$ the performance is slightly deteriorated with respect to the passive system. This performance deterioration can be prevented by choosing different values of $\alpha$ for both integrators in (13).

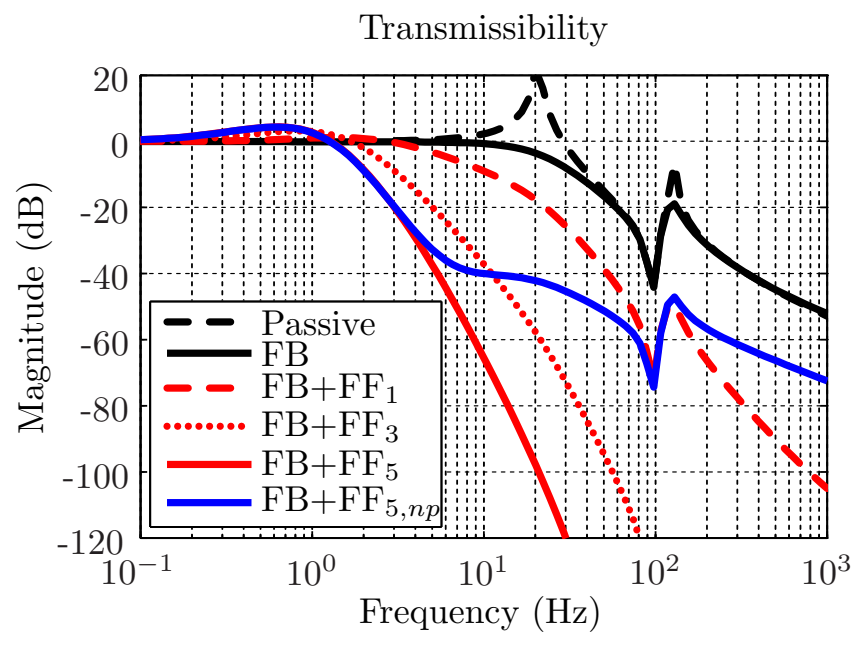

Fig. 3. Performance of the passive system, the feedback (FB) controlled system using (6), and the combined feedback plus fixed-gain feedforward (FF) controlled system using (6) and (13) with $n=1,3,5$. It also shows the performance for a $\mathrm{FB}+\mathrm{FF}$-controlled system with $n=5$ and non-perfect (np) estimates $\hat{k}_{1}=$ $0.99 k_{1}$ and $\hat{d}_{1}=0.9 d_{1}$.

Bode plots of $W_{1}, W_{3}$ and $W_{5}$ are compared with $W_{o p t}$ in Fig. 4. It is observed that increasing $n$ leads to less phase shift at the higher frequencies, and therefore a better performance for $\omega>\alpha$. However, increasing $n$ comes at the cost of a higher controller gain for frequencies lower than $\alpha$, which is undesired because of drift and actuator saturation. For example, the static gain for $W_{1}$ is $k_{1} / \alpha^{2}+$ $d_{1} / \alpha$, while the static gain for $W_{3}$ is $3 k_{1} / \alpha^{2}+d_{1} / \alpha$ and for $W_{5}$ it is $5 k_{1} / \alpha^{2}+d_{1} / \alpha$, hence a trade-off between the roll-off rate and the static controller gain. 
Bode diagram

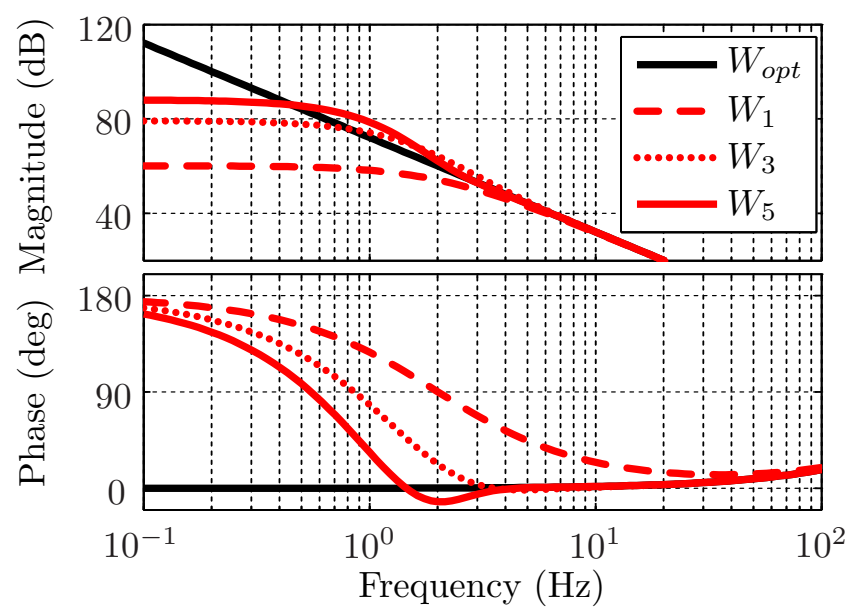

Fig. 4. Bode plots of the fixed-gain feedforward controllers. Increasing $n$ results in a trade-off: (a) a better approximation of the phase with respect to $W_{o p t}$, but (b) a higher static controller gain.

\subsection{Non-perfect estimates}

In practice, the estimates $\hat{k}_{1}$ and $\hat{d}_{1}$ will never be perfect, due to identification errors, non-linearities and timevarying behavior (e.g. caused by thermal effects). Having non-perfect estimates, the performance is limited by the error term $T_{\text {error }}$ in (14) at the higher frequencies. This is illustrated in Fig. 3, which shows the transmissibility in case of a $1 \%$ estimation error in the stiffness $\left(\hat{k}_{1}=0.99 k_{1}\right)$ and a $10 \%$ estimation error in the damping $\left(\hat{d}_{1}=0.9 d_{1}\right)$. For $\omega<k_{1} / d_{1}=2000 \mathrm{rad} / \mathrm{s}$, the performance is limited by the stiffness error, therefore the maximum vibration attenuation is $40 \mathrm{~dB}$ ( $1 \%$ of the original $T)$, while for $\omega>k_{1} / d_{1}$ the maximum vibration attenuation is $20 \mathrm{~dB}$ (10\% of the original $T$ ). This example illustrates the need for accurate parameter estimations.

\section{SELF-TUNING FEEDFORWARD CONTROL}

To obtain accurate values for $\hat{k}_{1}$ and $\hat{d}_{1}$, and thus minimizing performance limitations due to parameter estimation errors, the fixed-gain feedforward controller is redesigned as a self-tuning IIR filter with $n^{t h}$-order basis functions. The advantage of IIR filters over the widely used FIR filters, see e.g. Van der Poel (2010), is that filter poles are allowed at other locations than $z=0$. This makes sense, because from (13) it follows that poles are required. Moreover, the desired pole locations are known from (13). Therefore, the IIR filter poles can be fixed in the basis functions, resulting in a controller that is inherently stable because the poles cannot shift to unstable pole locations. Moreover, (13) is linear in the parameters $\hat{d}_{1}$ and $\hat{k}_{1}$. Therefore, minimization of a quadratic cost criterion to estimate $d_{1}$ and $k_{1}$ online leads to a convex adaptation problem with a unique global minimum. The preconditioned Filtered-Error LMS algorithm (Wesselink and Berkhoff (2008)) with residual noise shaping (Kuo and Tsai (1994)) is used for online parameter estimation.

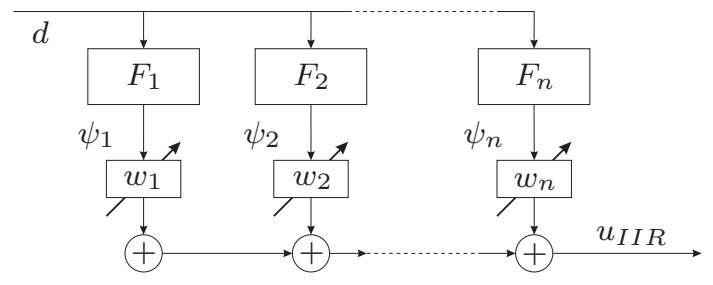

Fig. 5. Discrete-time IIR filter with self-tuning weights $w_{i}$

\subsection{Self-tuning IIR filter structure}

The IIR filter is depicted in Fig. 5. In the first step, $a_{0}$ is filtered by a vector of basis functions $F=\left[F_{1}, \ldots, F_{n}\right]$, resulting in $n$ signals collected in the vector $\psi=\left[\psi_{1}, \ldots, \psi_{n}\right]$. The signals $\psi_{i}$ are multiplied by self-tuning weights $w_{i}$, collected in $w=\left[w_{1}, \ldots, w_{n}\right]$. The sum of all weighted signals results in the IIR filter output $u_{I I R} . F_{i}$, with $i=1, \ldots, n$, are determined from the fixed-gain controller $W_{n}$, as will be explained in Section 5.3. The weights are determined using an update law,

$$
w(k+1)=w(k)-\frac{\mu(k)}{2}\left(\frac{\partial J(k)}{\partial w(k)}\right)^{T},
$$

with iteration step $k$ and adaptation rate $\mu(k)$. The gradient in (16) stems from the quadratic cost criterion

$$
J(k)=e^{\prime}(k)^{T} e^{\prime}(k) .
$$

Equation (17) describes the instantaneous squared filterederror rather than the mean squared filtered-error over a time interval. The filtered error $e^{\prime}=N(M \hat{S})^{-1} e(k)$ is obtained by filtering the measured error $e(k)$ by the inverse of the estimated secondary path $\hat{S}^{-1}$ and the preconditioning filter $M$ (to obtain the filtered-error LMS structure), and $N$ (to apply residual noise shaping). The filtered error is given by

$$
e^{\prime}(k)=N(\hat{S} M)^{-1}\left(P d(k)+S M\left[w^{T}(k) \psi(k)\right]\right),
$$

with input disturbance $d$, and $P, S$ as given in Fig. 2, $\psi$, $w$ as given in Fig. 5, and $M, N$ filters to be designed. It is generally not possible to find a closed-form expression for the gradient with respect to $w$. Therefore, slow adaptation of $w$ is assumed, such that the gradient in (16) can be written as

$$
\begin{aligned}
\frac{\partial J(k)}{\partial w(k)} & =\frac{\partial J(k)}{\partial e^{\prime}(k)} \cdot \frac{\partial e^{\prime}(k)}{\partial w(k)} \\
& \approx 2 e^{\prime}(k) \cdot N(\hat{S} M)^{-1} S M \psi(k) \\
& \approx 2 e^{\prime}(k) \cdot N \psi(k)
\end{aligned}
$$

In (21), it is assumed that $\hat{S}=S$. Recall from (3) that $S$ is assumed to have as many zeros as poles, such that $\hat{S}^{-1}$ is proper; modeling errors in $\hat{S}$ are discussed in section 5.3. Substitution of (21) in (16) gives the update law:

$$
w(k+1)=w(k)-\mu(k) \psi^{\prime}(k) e^{\prime}(k),
$$

with $\psi^{\prime}(k)=N \psi(k)$. Using a normalized step size, see Van der Poel (2010), we have

$$
\mu(k)=\frac{\bar{\mu}}{\epsilon+\left|\psi^{\prime}(k)\right|_{2}^{2}} .
$$

In (23), the adaptation rate is dependent on $\psi^{\prime}(k)$, such that instability of the adaptation process due to large input signals is prevented; $\epsilon>0$ is a small positive constant to prevent division by zero. The normalized adaptation rate $0<\bar{\mu}<2$, which is set by the user, 
must not be too large to prevent instability due to the approximations in (21).

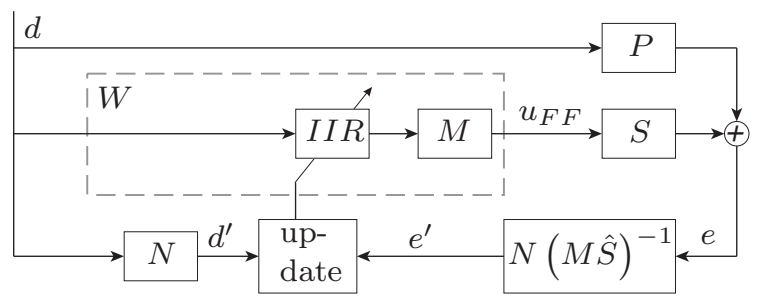

Fig. 6. Implementation of the self-tuning IIR filter.

Fig. 6 shows the implementation of the self-tuning IIR filter. The update block uses (22) to update the weights.

\subsection{Controller design}

The fixed-gain controller $W_{n}$ as given in (13) is redesigned as a self-tuning IIR filter, see (24). The parameters $\hat{d}_{1}$ and $\hat{k}_{1}$ in (13) have to be estimated online, so $w_{1}=\hat{d}_{1}$ and $w_{2}=\hat{k}_{1}$. The filters $M$ and $F_{i}$, with $i=1,2$, are discretetime equivalents of $M(s), F_{1}(s)$ and $F_{2}(s)$ as defined in

$$
W_{n, I I R}(s)=\underbrace{-\frac{1-L_{n}(s)}{s}}_{M(s)}(\underbrace{1}_{F_{1}(s)} w_{1}+\underbrace{\frac{1-L_{n}(s)}{s}}_{F_{2}(s)} w_{2}) \text {. }
$$

For use in a digital environment, all filters are discretized using the Tustin method with sampling frequency $6400 \mathrm{~Hz}$.

For the noise shaping filter $N$, the following design is used:

$$
N(s)=\left(\frac{s}{s+500}\right)\left(\frac{5000}{s+5000}\right)\left(\frac{s}{s+5 \alpha}\right)^{4} .
$$

The first-order high-pass filter with its pole at $s=$ $500 \mathrm{rad} / \mathrm{s}$ improves the convergence rate by making the frequency spectrum of $F_{2}(s) w_{2}$ flat. This makes sense, because the vibration isolator is a light-damped system, so $d_{1}$ (represented by $w_{1}$ ) will be small compared to $k_{1}$ (represented by $w_{2}$ ) and therefore the term $F_{2}(s) w_{2}$ is dominant at low frequencies $\omega<k_{1} / d_{1}$. The second term is a low-pass filter with a pole at $s=5000 \mathrm{rad} / \mathrm{s}$, this filter makes $N(M \hat{S})^{-1}$ proper. The fourth-order high-pass filter at $s=-5 \alpha \mathrm{rad} / \mathrm{s}$ removes the low-frequency content from the update signals. The low-frequency content is removed to prevent that the algorithm tries to compensate for low-frequency errors, which is not possible because of the tame integrators in (24). Without high-pass filtering, the estimations will be biased.

\subsection{Simulation results}

Simulation studies are performed for $n=1,3,5, \bar{\mu}=10^{-4}$, and $\epsilon$ such that its value is $0.1 \%$ of the RMS input power. The weights, all having an initial value of zero, are updated at every time step. Input $d$ is a floor acceleration with a white noise frequency spectrum. Fig. 7 shows the learning curves of the self-tuning algorithm. From the figure, it is clear that the algorithm converges to the minimum cost solution in all three cases where $\hat{S}=S$.

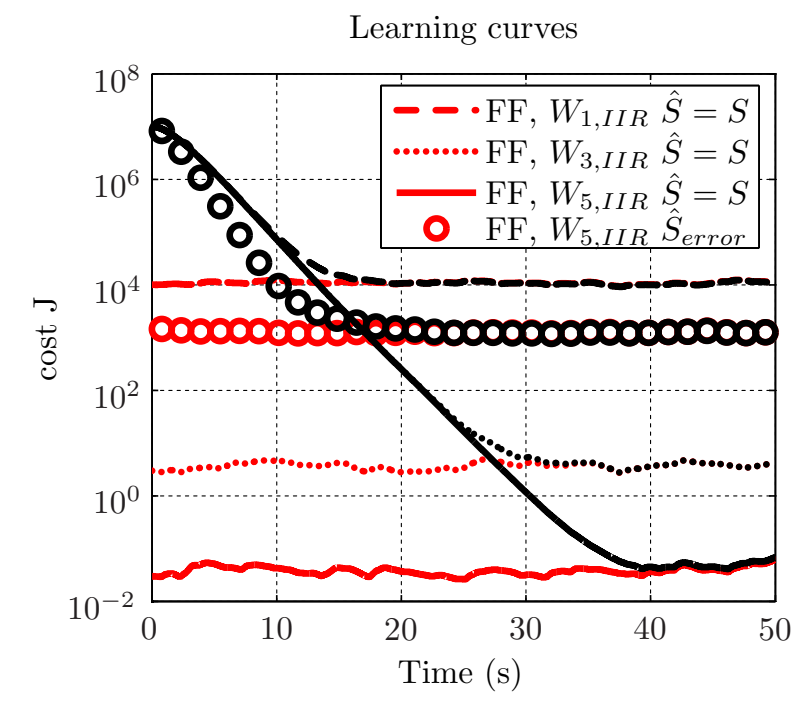

Fig. 7. Learning Curves, showing the convergence behavior of the IIR filter. The red lines indicate the minimum costs, corresponding to fixed-gain controllers with estimations $w_{1}=d_{1}$ and $w_{2}=k_{1}$. The black lines indicate the costs during the convergence process.

To investigate the sensitivity of the convergence algorithm for non-modeled high-frequency dynamics in $\hat{S}$, a fourth case is simulated in which an $800 \mathrm{~Hz}$ resonance with relative damping 0.07 is added to $S$, but not to $\hat{S}$. It appears that the self-tuning algorithm becomes unstable without modifying $N$. To re-stabilize the system in the presence of the $800 \mathrm{~Hz}$ resonance, a second-order lowpass filter at $s=500 \mathrm{rad} / \mathrm{s}$ is added to $N$, such that high-frequency content is filtered from the sensor signals. According to Bao and Panahi (2010), this is equivalent to virtually removing high-frequency content from $a_{0}$. As a result, excitation of the dynamics that destabilizes the converge process is not seen by the self-tuning controller. Note that the steady-state residual error is higher, because the resonance at $800 \mathrm{~Hz}$ is not compensated for by the controller.

When including feedback control, in addition to feedforward control, $\hat{S}$ is the estimate of the closed-loop secondary path, see Van der Poel (2010). Therefore, feedback control can be used to reduce modeling errors.

\section{EXPERIMENTAL RESULTS}

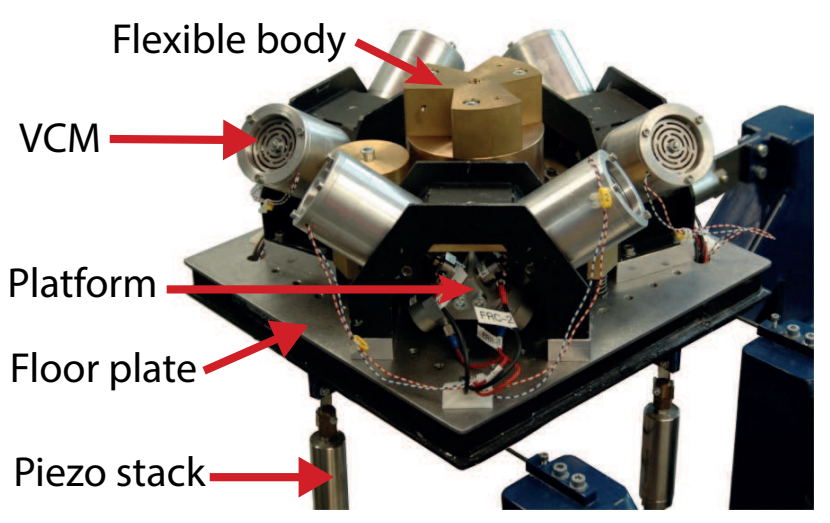

Fig. 8. Experimental setup. 
Fig. 8 shows the experimental setup used for validation purposes. The setup consists of a Stewart platform connected to six voice coil motors (VCMs) by wire springs. The VCMs are guided by circular leaf springs, which introduce six suspension modes into the system. The Stewart platform carries a flexible body that introduces three additional modes in the setup. The VCMs are controlled by a dSpace DSP system running at sampling frequency $f_{s}=$ $6400 \mathrm{~Hz}$. Both the floor plate and the Stewart platform contain six accelerometers. The floor plate can be excited in vertical direction by three piezo stacks providing random vibrations (white noise). The setup is designed such that the platform motion in vertical direction is almost decoupled from the other directions. In vertical direction, the passive system contains one suspension mode and one internal mode, see the measurements in Fig. 9.

The self-tuning feedforward controller from Section 5.3 is implemented on the DSP with $n \in\{1,3,5\}$ and $\alpha=2 \mathrm{~Hz}$, together with the feedback controller from (6). During the convergence process, a rigid-body estimation for $S$ including feedback controller (6) is used. To filter nonmodeled high-frequency dynamics, (25) is extended with a fourth-order low-pass filter at $500 \mathrm{rad} / \mathrm{s}$. From Fig. 9 it is observed that attenuation levels up to $40 \mathrm{~dB}$ are obtained with respect to the feedback-controlled system. At higher frequencies, active vibration isolation even deteriorates the performance due to the causal nature of the input disturbance measurement, see Heertjes et al. (2013). For frequencies higher than $400 \mathrm{~Hz}$, sensor noise dominates the error response. Furthermore, suspension modes show up at $18 \mathrm{~Hz}$ and $29 \mathrm{~Hz}$ due to non-perfect decoupling of the system. It is expected that a full Multiple-Input MultipleOutput (MIMO) feedforward controller would suppress these modes, this will be a topic for future research.

\section{Transmissibility}

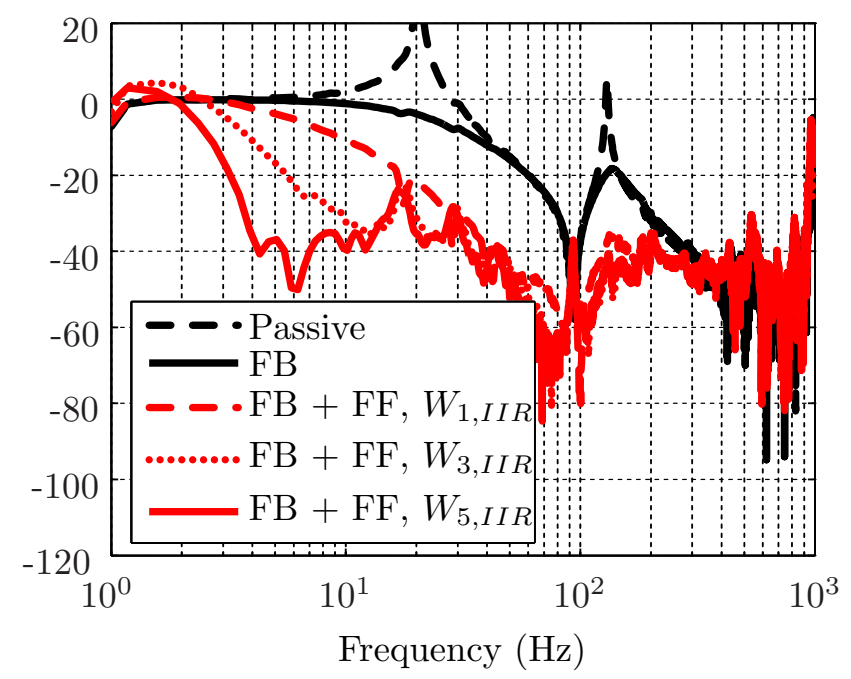

Fig. 9. Measured frequency response functions.

\section{CONCLUSIONS}

An effective self-tuning feedforward control strategy for the active vibration isolation of precision machinery from floor vibrations is presented and experimentally validated.
To prevent problems with drift and actuator saturation, the controller gain is limited for frequencies below $2 \mathrm{~Hz}$, while obtaining a good performance for higher frequencies. To reduce estimation errors, the feedforward controller is implemented as a self-tuning IIR filter. Simulations show that the self-tuning IIR filter converges to the optimal fixed-gain controller. An experimental validation of the self-tuning feedforward control strategy, combined with a skyhook feedback controller, shows that floor vibrations are suppressed up to $40 \mathrm{~dB}$ in a broad frequency range.

\section{ACKNOWLEDGEMENTS}

This research was financed by support of the Pieken in de Delta Program of the Dutch Ministry of Economic Affairs.

\section{REFERENCES}

Bao, H. and Panahi, I.M.S. (2010). A novel feedforward active noise control structure with spectrum-tuning for residual noise. Consumer Electronics, IEEE Transactions on, 56(4), 2093-2097.

Heertjes, M.F., Temizer, B., and Schneiders, M. (2013). Self-tuning in master-slave synchronization of highprecision stage systems. Control Engineering Practice, 21(12), 1706-1715.

Karnopp, D. and Trikha, A. (1969). Comparative study of optimization techniques for shock and vibration isolation. Transactions of American Society of Mechanical Engineers, Journal of Engineering for Industry, 91(4), 1128-1132.

Kuo, S.M. and Tsai, J. (1994). Residual noise shaping technique for active noise control systems. The Journal of the Acoustical Society of America, 95, 1665-1668.

Landau, I.D., Alma, M., and Airimitoaie, T.B. (2011). Adaptive feedforward compensation algorithms for active vibration control with mechanical coupling. Automatica, 47(10), $2185-2196$.

Tjepkema, D., van Dijk, J., and Soemers, H. (2011). Sensor fusion for active vibration isolation in precision equipment. Journal of Sound and Vibration, 331(4), $735-749$.

Van der Poel, G. (2010). An exploration of active hard mount vibration isolation for precision equipment. Ph.D. thesis, University of Twente, Enschede, The Netherlands.

Wesselink, J. and Berkhoff, A. (2008). Fast affine projections and the regularized modified filtered-error algorithm in multichannel active noise control. The Journal of the Acoustical Society of America, 124(2), 949-960.

Williamson, G.A. and Zimmermann, S. (1996). Globally convergent adaptive iir filters based on fixed pole locations. Signal Processing, IEEE Transactions on, 44(6), 1418-1427.

Yuan, J. (2007). Adaptive laguerre filters for active noise control. Applied Acoustics, 68(1), 86 - 96. doi: http://dx.doi.org/10.1016/j.apacoust.2006.01.009.

Zuo, L., Slotine, J.J., and Nayfeh, S. (2004). Experimental study of a novel adaptive controller for active vibration isolation. In Proceedings of the American Control Conference, 3863-3868. 